ESAIM: COCV 22 (2016) 1040-1053

DOI: $10.1051 / \mathrm{cocv} / 2016032$
ESAIM: Control, Optimisation and Calculus of Variations

www.esaim-cocv.org

\title{
LAGRANGIAN CONTROLLABILITY AT LOW REYNOLDS NUMBER*
}

\author{
O. GLASS $^{1}$ AND T. HORSIN ${ }^{2}$
}

\begin{abstract}
In this paper, we establish a result of Lagrangian controllability for a fluid at low Reynolds number, driven by the stationary Stokes equation. This amounts to the possibility of displacing a part of a fluid from one zone to another by suitably using a boundary control. This relies on a weak variant of the Runge-Walsh's theorem (on approximation of harmonic functions) concerning the Stokes equation. We give two variants of this result, one of which we believe to be better adapted to numerical simulations.
\end{abstract}

Mathematics Subject Classification. 76D07, 76D55, 35Q30, 34H05, 93B05, 35A35.

Received June 6, 2016. Accepted June 7, 2016.

\section{INTRODUCTION}

In this paper, we consider smooth solutions to the stationary Stokes equation in a bounded regular domain $\Omega$ of $\mathbb{R}^{N}$, with $N=2,3$ :

$$
\begin{gathered}
-\Delta u+\nabla p=0 \text { in } \Omega, \\
\operatorname{div} u=0 \text { in } \Omega, \\
\int_{\Omega} p \mathrm{~d} x=0 .
\end{gathered}
$$

This system is standard to describe incompressible and highly viscous fluids. Here $u: \Omega \rightarrow \mathbb{R}^{N}$ represents the velocity field, and $p: \Omega \rightarrow \mathbb{R}$ the pressure.

The main questions addressed in this paper are of two forms.

The first one is the problem of approximation of a solution of (1.1) defined on the domain $\Omega$, by solutions of the same system, but defined on a larger set. To be more precise, the question that we raise is the following: given $\Omega^{\prime}$ an open set in $\mathbb{R}^{N}$ such that $\bar{\Omega} \subset \Omega^{\prime}$ and $K$ a compact subset of $\Omega, k \in \mathbb{N}, \alpha \in(0,1)$, and given $(u, p)$ a regular solution of (1.1) and $\varepsilon>0$, is it possible to find $(\bar{u}, \bar{p})$ a solution of equations (1.1) on $\Omega^{\prime}$ such that

$$
\|(u, p)-(\bar{u}, \bar{p})\|_{C^{k, \alpha}(K)} \leq \varepsilon ?
$$

Keywords and phrases. Stokes system, controllability, Lagrangian controllability, Runge theorem.

* Dedicated to Jean-Michel Coron on the occasion of his 60th birthday.

1 CEREMADE, Université Paris-Dauphine \& CNRS, PSL, Place du Maréchal de Lattre de Tassigny, 75775 Paris cedex 16,

France. glass@ceremade.dauphine.fr

2 Conservatoire National des Arts et Métiers, M2N, Case 2D 5000, 292 rue Saint-Martin, 75003 Paris, France. 
This question, to which we give a partial positive answer, is related to the celebrated Runge theorem (see e.g. [22]) concerning the approximation of holomorphic functions by rational functions as well as to its extension by Walsh (see e.g. [12]) to the case of harmonic functions on an open set in $\mathbb{R}^{N}$. The former has been used in [13] to obtain a result of approximate Lagrangian controllability of the incompressible Euler equation in dimension 2, whereas the latter has been used in [14] to get a result in dimension 3. Both results can be considered as the cornerstone of the known approaches to Lagrangian controllability (though the complete method requires other technical results).

Our second main question here deals precisely with the Lagrangian controllability itself in the framework of the Stokes model. Given a fluid model, achieving the Lagrangian controllability between two subsets consists in being able to act on a given part of the domain in such a way that the resulting fluid flow maps one subset onto the other in a given time. Moreover, we require that during this time interval the fluid particles of the concerned subset do not leave the domain.

Let us be more specific on the problem under view. Let $\Omega$ be a smooth bounded domain, and $\Sigma$ an open nonempty part of its boundary. Here we consider (1.1) in quasi-static form, that is, the solution is timedependent even if the driving equation is stationary. Moreover, we consider the system controlled from $\Sigma$, that is, we assume that we can prescribe the boundary conditions on $\Sigma$. On $\partial \Omega \backslash \Sigma$, on the contrary, it is constrained by the standard Dirichlet boundary conditions. So we write the system as follows:

$$
\begin{gathered}
-\Delta u+\nabla p=0 \text { in }(0, T) \times \Omega, \\
\operatorname{div} u=0 \text { in }(0, T) \times \Omega, \\
\int_{\Omega} p \mathrm{~d} x=0, \text { on }(0, T), \\
u=0 \text { on }(0, T) \times(\partial \Omega \backslash \Sigma) .
\end{gathered}
$$

Given this fluid model, the Lagrangian controllability refers to the possibility, given two sets, to find a suitable control on $[0, T] \times \Sigma$, so that the flow associated to the solution $u$ sends the first set on the second one. There are natural constraints on the two 0sets: they should have the same area/volume (due to the incompressibility of the model), and we add a constraint on their form (namely, that they are smooth Jordan curves/surfaces). Moreover, one can relax this notion of Lagrangian controllability to the approximate Lagrangian controllability by asking the first set to be merely sent arbitrarily close to the target, rather than reaching it exactly. Of course one has to give a precise meaning to this "closeness"; it will be made clear in Theorem 2.6 below.

Note that system (1.2) is considered a good approximation of the standard Navier-Stokes system

$$
\begin{gathered}
\partial_{t} u+(u \cdot \nabla) u-\nu \Delta u+\nabla p=0 \\
\operatorname{div} u=0,
\end{gathered}
$$

when the Reynolds number Re tends to $0^{+}$, that is, when $\nu \rightarrow+\infty$, see for instance ([17], Chap. 9). This model has already been studied in the framework of controllability in different situations. In [23], a model of very small species moving using flagella like tails is studied. The localization of the action is used to model the fluid as in (1.2) but on a time dependent set. Let us also mention the works $[3,4,20]$, where the problem of swimming in a Stokes fluid which is considered to be quasi-static is considered.

The assumption of a very small Reynolds number means that the inertia is neglected compared to viscosity forces. We have already obtained Lagrangian controllability results in the opposite case of infinite Reynolds number which corresponds to the inviscid case driven by the Euler equation (see $[13,14]$ ). However in the intermediate case which would be described by Navier-Stokes equation (1.3), the Lagrangian controllability remains an open problem. 


\section{MAIN RESUlts}

The first main result of this paper concerns a partial extension of Walsh's theorem to the Stokes equation. It is an approximation result for solutions of equation (1.1). It is an adaptation of Walsh's theorem of harmonic approximation. For the reader's convenience, we recall Walsh's theorem or its following variant (see [12], Thm. 1.7).

Theorem 2.1 (Walsh, Gardiner). Let $\mathcal{O}$ be an open set in $\mathbb{R}^{N}$ and let $K \subset \mathcal{O}$ be a compact set in $\mathbb{R}^{N}$ such that that $\mathcal{O}^{*} \backslash K$ is connected, where $\mathcal{O}^{*}$ is the Alexandroff compactification of $\mathcal{O}$. Then, for each function $u$ which is harmonic on an open set containing $K$ and each $\varepsilon>0$, there is a harmonic function $v$ in $\mathcal{O}$ such that $\|v-u\|_{\infty}<\varepsilon$ on $K$.

Since we are considering the convergence of a sequence of harmonic functions, by elliptic regularity, the uniform convergence on $K$ actually involves the convergence in any $C^{k}$ norm on any compact set included in the topological interior of $K$. Hence, by extending a bit $K$ if necessary, we see that Theorem 2.1 is valid when replacing the $L^{\infty}$-norm with any $C^{k}$-norm. This fact we be used several times in the sequel.

The original situation in Walsh's theorem concerned the case when $\mathcal{O}=\mathbb{R}^{N}$ and $\mathbb{R}^{N} \backslash K$ is connected: in that case one can approximate on $K$ a harmonic function defined on a neighborhood of $K$ by harmonic polynomials.

Here we prove a variant of Theorem 2.1 for the Stokes equation, where the approximation is defined only on a bounded open set, not the whole space. Precisely, we establish the following.

Theorem 2.2. Let $K$ a compact set in $\mathbb{R}^{N}, N=2$ or 3 . Let $\mathcal{V}$ and $\Omega$ two bounded open sets such that $K \subset \mathcal{V}$, $\overline{\mathcal{V}} \subset \Omega$ and such that each connected component of $\mathbb{R}^{N} \backslash K$ that is not included in $\mathcal{V}$ contains an interior point of $\mathbb{R}^{N} \backslash \Omega$. Then for any solution $(u, p) \in C^{\infty}\left(\mathcal{V} ; \mathbb{R}^{N+1}\right)$ of the Stokes equation in $\mathcal{V}$ :

$$
\left\{\begin{array}{l}
-\Delta u+\nabla p=0 \\
\operatorname{div} u=0
\end{array}\right.
$$

for any $k \in \mathbb{N}$ and any $\varepsilon>0$ there exists $(\bar{u}, \bar{p}) \in C_{c}^{\infty}\left(\mathbb{R}^{N} ; \mathbb{R}^{N+1}\right)$ a solution of the Stokes equation in $\Omega$ :

$$
\left\{\begin{array}{l}
-\Delta \bar{u}+\nabla \bar{p}=0, \\
\operatorname{div} \bar{u}=0
\end{array}\right.
$$

and

$$
\|\bar{u}-u\|_{C^{k}(K)} \leq \varepsilon .
$$

Now we state our main application of this result, namely an approximate Lagrangian controllability result for the Stokes equation. For that, we first recall some topological and geometrical concepts.

Definition 2.3. A regular Jordan curve in $\mathbb{R}^{2}$ is the image of $\mathbb{S}^{1}$ by a diffeomorphism, and similarly a Jordan surface in $\mathbb{R}^{3}$ is the image of $\mathbb{S}^{2}$ by a diffeomorphism.

According to the Jordan's theorem and the Jordan-Brouwer's theorem, the complement of a Jordan curve/surface $\gamma$ in $\mathbb{R}^{N}, N=2$ or 3 , defines two connected components; the bounded one will be denoted $\operatorname{int}(\gamma)$. We emphasize that it is not to be mixed up with the topological interior of a subset $A$, which we denote $A^{\circ}$. The exterior normal to Jordan curves/surfaces inside $\Omega$ will be denoted by $\nu$ whereas $n$ will be preferred in the case of the boundary of $\Omega$.

Also, we recall the following definitions.

Definition 2.4. Two Jordan curves $\gamma_{0}$ and $\gamma_{1}$ are said to be homotopic in $\Omega$, if there exists a continuous map $h:[0,1] \times \mathbb{S}^{1} \rightarrow \Omega$ such that $h(0, \cdot)$ is a parameterization of $\gamma_{0}$ and $h(1, \cdot)$ is a parameterization of $\gamma_{1}$. 
Definition 2.5. Two Jordan surfaces $\gamma_{0}$ and $\gamma_{1}$ embedded in $\mathbb{R}^{3}$ are said to be isotopic in $\Omega$, if there exists a continuous map $h:[0,1] \times \mathbb{S}^{2} \rightarrow \Omega$ (called isotopy) such that $h(0)=\gamma_{0}, h(1)=\gamma_{1}$ and for each $t \in$ $[0,1], h(t, \cdot)$ is an homeomorphism of $\mathbb{S}^{2}$ into its image. When, for some $k \in \mathbb{N} \backslash\{0\}$, this homeomorphism is a $C^{k}$-diffeomorphism, $h$ will be said to be a $C^{k}$-isotopy, or, when $k=\infty$, a smooth isotopy.

Note that when $N=2$, two homotopic Jordan curves in $\Omega$ are in fact isotopic in $\Omega$, see [10]. We will say that a surface is homotopic to a point when it is homotopic to a constant map.

We will also use the following notation for a flow: given a suitably regular vector field $u$, we denote by $\phi^{u}$ the flow of $u$, defined (when possible) by

$$
\partial_{t} \phi^{u}(t, s, x)=u\left(t, \phi^{u}(t, s, x)\right) \text { and } \phi^{u}(s, s, x)=x .
$$

Our result is then as follows.

Theorem 2.6. Let $\Omega$ be a smooth bounded open connected set in $\mathbb{R}^{N}$ and $\Sigma$ a nonempty open part of $\partial \Omega$. If $\gamma_{0}$ and $\gamma_{1}$ are smooth Jordan curves/surface in $\Omega$, isotopic in $\Omega$, then, for any $\varepsilon>0, k \in \mathbb{N}^{*}, \alpha \in(0,1)$, there exists a solution $(u, p) \in C^{\infty}\left([0,1] \times \bar{\Omega} ; \mathbb{R}^{N+1}\right)$ of $(1.2)$ such that

$$
\begin{array}{r}
\left\|\phi^{u}\left(1,0, \gamma_{0}\right)-\gamma_{1}\right\|_{C^{k, \alpha}\left(\mathbb{S}^{N-1}\right)} \leq \varepsilon, \\
\forall t \in[0,1], \phi^{u}\left(t, 0, \gamma_{0}\right) \subset \Omega .
\end{array}
$$

What we mean by (2.5a), is that we can find a parameterization of $\gamma_{0}$ and $\gamma_{1}$ such that (2.5a) holds. Naturally, for this quasi-static model, the time of controllability $T$ plays no role, and without loss of generality one can consider the problem when $T=1$ by a simple change of time variable.

Remark 2.7. If $\gamma_{0}$ is a real analytic surface, and if for all $t \in[0,1], \phi^{u}\left(t, 0, \gamma_{0}\right) \subset \Omega$, then by using the regularity of the solutions of the Stokes system, one deduces that for any $t \in[0,1], \phi^{u}\left(t, 0, \gamma_{0}\right)$ is also real analytic for anyt. Thus exact Lagrangian controllability does not hold in general in this framework (for example by choosing $\gamma_{1}$ smooth but not analytic).

The structure of the rest of the paper is as follows. In Section 3, we establish our Runge-Walsh type theorem for the Stokes equation, Theorem 2.2. Then in Section 4 we state and prove a variant to Theorem 2.2, which we believe is more suited to numerical approximation. In Section 5 we prove our main result on Lagrangian controllability, that is Theorem 2.6. We conclude the paper in Section 6 with a final remark.

\section{Proof of Theorem 2.2}

In this section, we establish Theorem 2.2.

Step 0. Geometrical simplification. In a first step, we show how to reduce to the case where $K$ and $\mathcal{V}$ have smooth boundaries and $\Omega$ is of the form $B(0, R) \backslash \cup_{i=1}^{n} B\left(A_{i}, r_{i}\right)$ with large $R>0$ and small $r_{i}>0$.

- Since $\Omega$ is bounded, it is included in some ball $B(0, R / 2), R$ large enough. In each connected component of $B(0, R) \backslash K$ not included in $\mathcal{V}$, we pick a unique point $A_{i}$ in the topological interior of $\mathbb{R}^{N} \backslash \Omega$ (which is clearly possible due to the assumption). Consequently there exists $r_{i}>0$ such that $\bar{B}\left(A_{i}, r_{i}\right) \subset \mathbb{R}^{N} \backslash \Omega$. In particular, note that $B\left(A_{i}, r_{i}\right)$ does not intersect $\mathcal{V}$. Of course, since each of such connected component contains a point with rational coordinates, the family $\left(A_{i}\right)$ is at most countable. Clearly one has $\Omega \subset \tilde{\Omega}:=$ $B(0, R) \backslash \cup_{i} \bar{B}\left(A_{i}, r_{i}\right)$. Finally, we add to $K$ all the connected components of $B(0, R) \backslash K$ included in $\mathcal{V}$ : call $\tilde{K}$ the resulting set which is still compact (it is bounded and contains its boundary). Now if we can establish the result on $\tilde{K}, \mathcal{V}$ and $\tilde{\Omega}$ (that is, starting from a solution in $\mathcal{V}$, approximate it on $\tilde{K}$ by a solution in $\tilde{\Omega}$ ), it is all the more true on $K, \mathcal{V}$ and $\Omega$. 


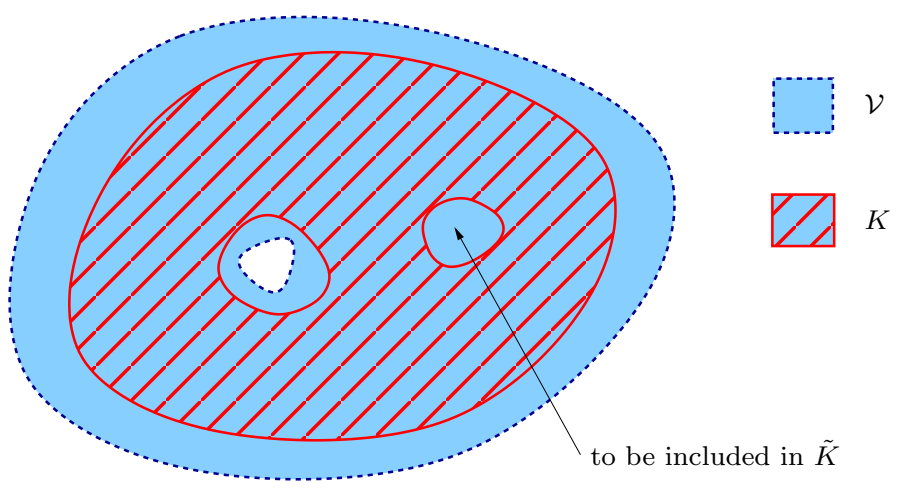

Figure 1. The sets $K$ and $\mathcal{V}$.

- We claim that $B(0, R) \backslash \tilde{K}$ has a finite number of connected components. If not, consider the subsequence of the points $A_{i}$ associated to the connected components in $B(0, R) \backslash \tilde{K}$. Write it $\left(A_{i}\right)_{i \in \mathbb{N}}$ again. Then up to a subsequence that we still denote $\left(A_{i}\right)$, one has $A_{i} \rightarrow A$ as $i \rightarrow+\infty$. Then $A$ cannot belong to a connected component of $B(0, R) \backslash \tilde{K}$, since otherwise, there would be a whole ball around $A$ included in this connected component, and each of these connected components is only visited once by the sequence $A_{i}$. It follows that $A \in \tilde{K} \subset \mathcal{V}$. This means that for $i$ large, $A_{i} \in \mathcal{V}$ which contradicts the fact that $B\left(A_{i}, r_{i}\right)$ does not meet $\mathcal{V}$. We call $A_{1}, \ldots, A_{n}$ the remaining points $A_{i}$ corresponding to the connected components of $B(0, R) \backslash \tilde{K}$.

- Now by the Whitney extension theorem, there exists a function $\varphi \in C^{\infty}(\bar{B}(0, R) ; \mathbb{R})$ such that $\tilde{K}=\varphi^{-1}(\{0\})$. Replacing $\varphi$ by $\varphi^{2}$ if necessary, we may assume that $\varphi \geq 0$. Due to the compactness of $\tilde{K}$, we have for some $c>0$ :

$$
\varphi \geq c \text { in } \bar{B}(0, R) \backslash \mathcal{V} .
$$

Consider $\delta \in[0, c)$. Due to Sard's theorem, there exists regular values $\lambda, \mu$ and $\nu$ of the function $\varphi$ respectively in $[\delta / 10, \delta / 5],[3 \delta / 10,2 \delta / 5]$ and $[\delta / 2,3 \delta / 5]$. Call $K_{\delta}:=\varphi^{-1}([0, \lambda]), \mathcal{V}_{\delta}^{1}:=\varphi^{-1}([0, \mu))$ and $\mathcal{V}_{\delta}:=\varphi^{-1}([0, \nu))$. All the three subsets have consequently smooth boundaries. Furthermore, reducing $\delta$ if necessary, we can ensure that $\cup_{i=1}^{n} \bar{B}\left(A_{i}, r_{i}\right) \cap \mathcal{V}_{\delta}=\emptyset$. Moreover:

- if there are connected components of $K_{\delta}$ that do not intersect $K$, remove them from $K_{\delta}$,

- if there are connected components of $B(0, R) \backslash \mathcal{V}_{\delta}^{1}$ and $B(0, R) \backslash \mathcal{V}_{\delta}$ that do not contain a point $A_{i}$, add them to $\mathcal{V}_{\delta}^{1}\left(\right.$ resp. $\left.\mathcal{V}_{\delta}\right)$.

Note that since $\mathcal{V}_{\delta}^{1}$ and $\mathcal{V}_{\delta}$ are included in $\mathcal{V}$, a connected component of $B(0, R) \backslash \mathcal{V}_{\delta}^{1}$ (resp. $\left.B(0, R) \backslash \mathcal{V}_{\delta}\right)$ either contains a connected component of $B(0, R) \backslash \mathcal{V}$ (and hence a point $\left.A_{i}\right)$ or is included in $\mathcal{V}$. Consequently $\mathcal{V}_{\delta}^{1}$ and $\mathcal{V}_{\delta}$ obtained in this way are included in $\mathcal{V}$.

Now if we can establish the result on $K_{\delta}, \mathcal{V}_{\delta}$ and $\hat{\Omega}:=B(0, R) \backslash \cup_{i=1}^{n} \bar{B}\left(A_{i}, r_{i}\right)$, it is all the more true on $K$, $\mathcal{V}$ and $\Omega$. From now on, we write $K, \mathcal{V}$ and $\Omega$ for $K_{\delta}, \mathcal{V}_{\delta}$ and $\hat{\Omega}$, to simplify the notation. Note in passing that we have obtained $\mathcal{V}_{1}$ (the new notation for $\mathcal{V}_{\delta}^{1}$ ) with smooth boundary so that

$$
K \subset \mathcal{V}_{1} \subset \overline{\mathcal{V}_{1}} \subset \mathcal{V} \subset \overline{\mathcal{V}} \subset \Omega
$$

This ends our preliminary step of geometrical simplification.

Now let us go back to the proof of Theorem 2.2. We consider $(u, p)$ a smooth solution of $(2.2)$ on $\mathcal{V}$, and want a solution of (2.2) on $\Omega$ suitably approximating $u$ on $K$. We follow several consecutive steps. In the sequel, $C>0$ is a constant that may change from line to line and depends on the geometry, but not on the function $u$ nor on $\varepsilon$. 
Step 1. First we introduce an extension $\tilde{p} \in C_{c}^{\infty}(\Omega ; \mathbb{R})$ of $p_{\mid \overline{\mathcal{V}}}$. Next we define

$$
\hat{u}:=u-\nabla \Delta^{-1} \tilde{p} \text { in } \mathcal{V}
$$

where $\Delta^{-1}:=* * G$ is the convolution operator with the fundamental solution associated to the Laplacian, that is,

$$
G(x)=\frac{1}{2 \pi} \ln |x| \text { if } N=2, \quad G(x)=-\frac{1}{4 \pi|x|} \text { if } N=3 .
$$

Then one can easily check that

$$
\operatorname{curl} \hat{u}=\operatorname{curl} u \text { in } \mathcal{V}, \quad \Delta \hat{u}=0 \text { in } \mathcal{V} .
$$

Let $\gamma_{1}, \ldots, \gamma_{g}$ a (minimal) family of smooth loops generating the fundamental group of $\mathcal{V}_{1}$. One has moreover

$$
\oint_{\gamma_{i}}(\hat{u}-u) \cdot \tau=0, \quad \forall i=1, \ldots, g .
$$

Step 2. Next we use Walsh's theorem on $\hat{u}$ which is harmonic in $\mathcal{V}$. We have points $A_{1}, \ldots, A_{n}$ outside $\Omega$ in each connected component of $\mathbb{R}^{N} \backslash \mathcal{V}_{1}$. Hence we obtain a harmonic function $\tilde{u}$, defined in $\mathbb{R}^{N} \backslash\left\{A_{1}, \ldots, A_{n}\right\}$ and such that, given $\alpha \in(0,1)$ :

$$
\begin{array}{r}
\Delta \tilde{u}=0 \text { in } \mathbb{R}^{N} \backslash\left\{A_{1}, \ldots, A_{n}\right\}, \\
\|\hat{u}-\tilde{u}\|_{C^{k+1, \alpha}\left(\overline{\mathcal{V}_{1}}\right)} \leq \varepsilon .
\end{array}
$$

In particular we infer from (3.1)-(3.3) that for some $C>0$,

$$
\left|\oint_{\gamma_{i}}(u-\tilde{u}) \cdot \tau\right| \leq C \varepsilon \text { and }\|\operatorname{curl} u-\operatorname{curl} \tilde{u}\|_{C^{k, \alpha}\left(\overline{\mathcal{V}_{1}}\right)} \leq C \varepsilon .
$$

Step 3. Now we extend $\operatorname{curl}(u-\tilde{u})_{\mid \overline{\mathcal{V}_{1}}}$ to a function $w \in C_{c}^{k, \alpha}\left(\mathbb{R}^{N}\right)$ such that

$$
\|w\|_{C_{c}^{k, \alpha}\left(\mathbb{R}^{N}\right)} \leq C \varepsilon .
$$

Now, for instance by using Biot-Savart's formula (see e.g. [5] for a historical reference), there exists $v \in$ $C^{k+1, \alpha}\left(\mathbb{R}^{N}\right)$ such that for a constant $C>0$ depending on $R$ :

$$
\operatorname{curl} v=w, \operatorname{div} v=0, \quad\|v\|_{C^{k+1, \alpha}(\bar{B}(0, R))} \leq C \varepsilon .
$$

Then $\operatorname{curl} v=\operatorname{curl}(u-\tilde{u})$ on $\mathcal{V}_{1}$, so one has

$$
v=u-\tilde{u}+\nabla \theta+H
$$

for some $\theta \in C^{k+2, \alpha}\left(\mathcal{V}_{1} ; \mathbb{R}\right)$ and $H$ some function in the first de Rham cohomology space of $\mathcal{V}_{1}$. We recall that the first de Rham cohomology space of $\mathcal{V}_{1}$ is the quotient space of the space of curl-free vector fields by gradients vector fields. This vector space is of finite dimension $g$ and can be represented in terms of vector fields by

$$
\mathcal{H}^{1}\left(\mathcal{V}_{1}\right):=\left\{u \in C^{\infty}\left(\overline{\mathcal{V}}_{1} ; \mathbb{R}^{N}\right) / \operatorname{curl} u=0 \text { and } \operatorname{div} u=0 \text { in } \mathcal{V}_{1}, u \cdot n=0 \text { on } \partial \mathcal{V}_{1}\right\}
$$

Moreover the map $\mathcal{I}: \mathcal{H}^{1}\left(\mathcal{V}_{1}\right) \rightarrow \mathbb{R}^{g}, \quad H \mapsto\left(\oint_{\gamma_{i}} H \cdot \tau\right)_{i=1, \ldots, g}$ is well-defined (the result is independent of the representative in the class) and is an isomorphism. See for instance ([24], Appendix I) or [15]. We use this function space as follows: any curl-free vector field in $\mathcal{V}_{1}$ is the sum of a gradient and an element of $\mathcal{H}^{1}\left(\mathcal{V}_{1}\right)$. 
We extend $\theta$ to some function in $C_{c}^{k+2, \alpha}\left(\mathbb{R}^{N} ; \mathbb{R}\right)$ arbitrarily. In particular, we have

$$
\|u-\tilde{u}+\nabla \theta+H\|_{C^{k+1, \alpha}\left(\mathcal{V}_{1}\right)} \leq C \varepsilon .
$$

From (3.6) and (3.7) we deduce that for all $i=1, \ldots, g$, one has

$$
\left|\oint_{\gamma_{i}} H \cdot \tau\right| \leq C \varepsilon
$$

Since $\mathcal{I}$ is an isomorphism we deduce finally that

$$
\|H\|_{C^{k+1, \alpha}\left(\mathcal{V}_{1}\right)} \leq C \varepsilon
$$

and consequently that

$$
\|u-\tilde{u}+\nabla \theta\|_{C^{k+1, \alpha}\left(\mathcal{V}_{1}\right)} \leq C \varepsilon
$$

Step 4. Now we claim that there exists $\beta \in C^{k+1, \alpha}(\Omega)$ such that

$$
\begin{gathered}
\|\nabla \beta\|_{C^{k, \alpha}(K)} \leq C \varepsilon, \\
\Delta \beta=\operatorname{div}(\tilde{u}-\nabla \theta) \text { in } \Omega .
\end{gathered}
$$

To prove this we proceed as in Step 3 with div replacing curl. Namely, we extend $\operatorname{div}(\tilde{u}-\nabla \theta)_{\mid \overline{\mathcal{V}_{1}}}$ to some function $\rho \in C_{c}^{k, \alpha}\left(\mathbb{R}^{N}\right)$ in such a way that

$$
\|\rho\|_{C_{c}^{k, \alpha}\left(\mathbb{R}^{N}\right)} \leq C\left\|\operatorname{div}(\tilde{u}-\nabla \theta)_{\overline{\mathcal{V}_{1}}}\right\|_{C^{k, \alpha}\left(\mathcal{V}_{1}\right)} .
$$

Note that due to (3.9) and since $u$ satisfies $(2.1)$ on $\mathcal{V}$, the right hand side is of order $\mathcal{O}(\varepsilon)$. Now there is a vector field $v_{\rho} \in C^{k+1, \alpha}(B(0, R))$ such that

$$
\operatorname{div} v_{\rho}=\rho \text { in } B(0, R) \text { and }\left\|v_{\rho}\right\|_{C^{k+1, \alpha}(B(0, R))} \leq C\|\rho\|_{C_{c}^{k, \alpha}\left(\mathbb{R}^{N}\right)} \leq C \varepsilon
$$

Notice that

$$
\operatorname{div}\left(\tilde{u}-\nabla \theta-v_{\rho}\right)=0 \text { in } \mathcal{V}_{1} .
$$

We suppose here $N=3$. One can adapt the proof mutatis mutandis to $N=2$.

Call $\Gamma_{0}, \Gamma_{1}, \ldots, \Gamma_{M}$ the various connected components of $\partial \mathcal{V}_{1}, \Gamma_{0}$ being the exterior one. Taking (3.12) into account and due to the fact that each connected component of $\mathbb{R}^{N} \backslash \mathcal{V}_{1}$ meets at least a point $A_{i}$, we deduce that there exist $\lambda_{1}, \ldots, \lambda_{M}$ so that

$$
\forall i=1, \ldots, M, \quad \int_{\Gamma_{i}}\left(\tilde{u}-\nabla \theta-v_{\rho}-\sum_{j=1}^{M} \lambda_{j} \frac{x-A_{j}}{\left|x-A_{j}\right|^{3}}\right) \cdot n=0 .
$$

We write

$$
h:=\sum_{j=1}^{M} \lambda_{j} \frac{x-A_{j}}{\left|x-A_{j}\right|^{3}}
$$

The functions defining $h$ are related to the second de Rham cohomology space of $\mathcal{V}_{1}$, denoted $\mathcal{H}^{2}\left(\mathcal{V}_{1}\right)$, which is a finite dimensional space and which can also be described as in ([24], Appendix I). We recall that the second de Rham cohomology space of $\mathcal{V}_{1}$ is the quotient space of the space of div-free vector fields by vector fields that can be put in the form curl $A$. It has dimension $M$ and is for instance generated by the family of functions 
$x \mapsto \frac{x-A_{j}}{\left|x-A_{j}\right|^{3}}, j=1, \ldots, M$. We use it to express that any divergence-free vector field can be written as a sum of a curl and an element of $\mathcal{H}^{2}\left(\mathcal{V}_{1}\right)$. Now on $\mathcal{V}_{1}$, one has $\operatorname{div}\left(\tilde{u}-\nabla \theta-v_{\rho}-h\right)=0$ and

$$
\int_{\Gamma}\left(\tilde{u}-\nabla \theta-v_{\rho}-h\right) \cdot n=0
$$

on any closed surface $\Gamma \subset \mathcal{V}_{1}$. It follows that that $\tilde{u}-\nabla \theta-v_{\rho}$ can be written in the form

$$
\tilde{u}-\nabla \theta-v_{\rho}=\operatorname{curl} B+h \text { in } \mathcal{V}_{1},
$$

for some $B \in C^{k+2, \alpha}\left(\mathcal{V}_{1}\right)$. In particular one has

$$
\|\tilde{u}-\nabla \theta-\operatorname{curl} B-h\|_{C^{k+1, \alpha}\left(\mathcal{V}_{1}\right)}=\left\|v_{\rho}\right\|_{C^{k+1, \alpha}(B(0, R))} \leq C \varepsilon .
$$

Now we extend $(\tilde{u}-\nabla \theta-\operatorname{curl} B-h)_{\mid \mathcal{V}_{1}}$ into some $\hat{U} \in C^{k+1, \alpha}\left(\Omega ; \mathbb{R}^{3}\right)$ and $B$ in some $\tilde{B} \in C_{c}^{k+2, \alpha}\left(B(0, R) ; \mathbb{R}^{3}\right)$ in such a way that

$$
\|\hat{U}\|_{C^{k+1, \alpha}(\Omega)} \leq C\|\tilde{u}-\nabla \theta-\operatorname{curl} B-h\|_{C^{k+1, \alpha}\left(\mathcal{V}_{1}\right)} .
$$

We define

$$
\tilde{U}:=\hat{U}+\operatorname{curl} \tilde{B}+h \text { in } \Omega .
$$

We introduce $\tilde{q}$ as the solution in $\Omega$ of

$$
-\Delta \tilde{q}=\operatorname{div} \tilde{U}=\operatorname{div} \hat{U} \text { in } \Omega, \quad \tilde{q}=0 \text { on } \partial \Omega,
$$

and $q$ as the solution in $\Omega$ of

$$
-\Delta q=\operatorname{div}(\tilde{u}-\nabla \theta) \text { in } \Omega, \quad \tilde{q}=0 \text { on } \partial \Omega .
$$

Note that thanks to (3.14) and (3.15), we have

$$
\|\tilde{q}\|_{C^{k+1, \alpha}(\Omega)} \leq C \varepsilon .
$$

Clearly, $\tilde{q}-q$ is harmonic on $\mathcal{V}_{1}$, so by Walsh's theorem one can find some harmonic function $\psi$ in $\mathbb{R}^{N} \backslash$ $\left\{A_{1}, \ldots, A_{n}\right\}$ such that

$$
\|\tilde{q}-q-\psi\|_{C^{k+1, \alpha}(K)} \leq \varepsilon .
$$

We let

$$
\beta:=-q-\psi \text {. }
$$

One can easily check that (3.11) holds, and (3.10) is a consequence of (3.16), (3.17) and

$$
\|\beta\|_{C^{k+1, \alpha}(K)} \leq\|\tilde{q}-q-\psi\|_{C^{k+1, \alpha}(K)}+\|\tilde{q}\|_{C^{k+1, \alpha}(K)} .
$$

Conclusion. We define

$$
\bar{u}:=\tilde{u}-\nabla \theta-\nabla \beta .
$$

Due to (3.9) and (3.10) one has

$$
\|u-\bar{u}\|_{C^{k, \alpha}(K)} \leq C \varepsilon .
$$

Moreover

$$
\operatorname{div} \bar{u}=0 \text { in } \Omega
$$

follows from (3.11). Finally one has

$$
\Delta \bar{u}=-\nabla(\Delta(\theta+\beta)) \text { in } \Omega,
$$

thanks to (3.4). This concludes the proof of Theorem 2.2. 


\section{An alternative approach}

The above proof relies on the aforementioned Walsh theorem (Thm. 2.1). The proof of Walsh's theorem is not really constructive; at least it seems difficult to propose a numerical scheme which could be inherited from the proof. To take this into account, we propose another strategy for the subsequent application, based on a density argument which we hope can be used more easily for an approximation scheme. Of course, this density result can also be proved by using Theorem 2.2.

We first introduce some notations. Given a smooth Jordan curve/surface $\gamma$ we define

$$
H_{m}^{1 / 2}(\gamma):=\left\{\phi \in H^{1 / 2}(\gamma)^{N}, \int_{\gamma} \phi \cdot n \mathrm{~d} \sigma=0\right\} .
$$

For $\Sigma$ a nonempty open part of the boundary of some regular open set $U$, we moreover consider

$$
H_{m}^{1 / 2}(\partial U ; \Sigma):=\left\{\phi \in H^{1 / 2}(\partial U)^{N}, \operatorname{Supp} \phi \subset \Sigma, \int_{\Sigma} \phi \cdot n \mathrm{~d} \sigma=0\right\} .
$$

The density result that we prove is the following.

Theorem 4.1. Let $\Omega$ a smooth bounded domain, and let $\Sigma$ an open nonempty part of its boundary. Assume that $\gamma$ is a $C^{\infty}$ Jordan surface included in $\Omega$, then the set

$$
\left\{u_{\mid \gamma},(u, p) \text { is a solution of }(1.2) \text { such that } u_{\mid \partial \Omega} \in H_{m}^{1 / 2}(\partial U ; \Sigma)\right\},
$$

is dense in $H_{m}^{1 / 2}(\gamma)$.

Proof of Theorem 4.1. We first assume that $\Sigma \cap{ }^{c} \operatorname{int}(\gamma) \neq \emptyset$. We recall that int $(\gamma)$ denotes the interior of the Jordan surface $\gamma$ in the sense of Jordan's theorem (and not the topological interior). Consider some $T \in$ $H^{-1 / 2}(\gamma)^{N}$. To such $T$ we associate $T_{\gamma} \in \mathcal{D}^{\prime}(\Omega)$ by

$$
\left\langle T_{\gamma}, \varphi\right\rangle_{\mathcal{D}^{\prime}(\Omega) \times \mathcal{D}(\Omega)}:=\left\langle T, \varphi_{\mid \gamma}\right\rangle_{H^{-1 / 2}(\gamma) \times H^{1 / 2}(\gamma)} .
$$

It is not difficult to see that $T_{\gamma}$ is continuous on $H_{0}^{1}(\Omega)^{N}$, so that $T_{\gamma} \in H^{-1}(\Omega)^{N}$. Hence we can introduce $(v, q) \in H_{0}^{1}(\Omega)^{N} \times L^{2}(\Omega)$ the solution of

$$
\begin{aligned}
-\Delta v+\nabla q & =T_{\gamma} \text { in } \Omega, \\
\operatorname{div} v & =0 \text { in } \Omega, \\
v & =0 \text { on } \partial \Omega, \\
\int_{\Omega} q \mathrm{~d} x & =0 .
\end{aligned}
$$

Consider now $u \in H^{1}(\Omega)^{N}$ a solution of (1.1) with $u_{\mid \partial \Omega} \in H_{m}^{1 / 2}(\partial \Omega ; \Sigma)$. We perform the following computations:

$$
\begin{aligned}
T\left(u_{\mid \gamma}\right) & =\int_{\Omega}(-\Delta v+\nabla q) \cdot u \mathrm{~d} x \\
& =\int_{\Omega}-\Delta u \cdot v \mathrm{~d} x+\int_{\partial \Omega}\left(-\frac{\partial v}{\partial n}+q n\right) \cdot u \mathrm{~d} \sigma \\
& =\int_{\partial \Omega}\left(-\frac{\partial v}{\partial n}+q n\right) \cdot u \mathrm{~d} \sigma
\end{aligned}
$$

We remark that the term $-\frac{\partial v}{\partial n}+q n$ in the right hand side of the last identity is defined in a classical way due to the local regularity of $(v, q)$ away from $\gamma$. 
Now assume that there exists some $T \in H_{m}^{1 / 2}(\gamma)^{\prime}$ such that $T\left(u_{\mid \gamma}\right)=0$ for any $(u, p)$ solution of (1.1) with $u_{\mid \partial \Omega} \in H_{m}^{1 / 2}(\partial \Omega ; \Sigma)$. Of course, $T$ can be extended as a continuous linear form on $H^{1 / 2}(\gamma)^{N}$, so $T$ belongs to $H^{-1 / 2}(\gamma)^{N}$. From (4.2) we can deduce that $-\frac{\partial v}{\partial n}+q n=0$ on $\Sigma$. Since moreover $v \in H_{0}^{1}(\Omega)^{N}$, by using the unique continuation property for the Stokes equation (see $[7,11]$ ) we deduce that $v=0$ and thus $q=0$ in $\Omega \backslash \operatorname{int}(\gamma)$.

Since $v \in H_{0}^{1}(\Omega)^{N}$ and $q \in L^{2}(\Omega)$, we infer that $v_{\mid \operatorname{int}(\gamma) \cap \Omega} \in H_{0}^{1}(\operatorname{int}(\gamma) \cap \Omega)$ and $q_{\mid \operatorname{int}(\gamma) \cap \Omega} \in L^{2}(\operatorname{int}(\gamma) \cap \Omega)$. Since moreover one has

$$
-\Delta v+\nabla q=0 \text { and } \operatorname{div} v=0 \text { in } \mathcal{D}^{\prime}(\operatorname{int}(\gamma) \cap \Omega),
$$

we can thus infer by uniqueness that $v=0$ and $q$ is constant in $\operatorname{int}(\gamma) \cap \Omega$. Now if $q$ is a non null constant in $\operatorname{int}(\gamma) \cap \Omega$ while $q=0$ in $\Omega \backslash \operatorname{int}(\gamma)$, this leads to the following form of $T$ :

$$
T(w)=c \int_{\gamma} w \cdot \nu \mathrm{d} \sigma
$$

which means that $T=0$ as an element of $H_{m}^{1 / 2}(\gamma)^{\prime}$. This establishes the density claimed in Theorem 4.1.

To treat the case where $\Sigma \subset \operatorname{int}(\gamma)$, we just have to exchange the role played by $\operatorname{int}(\gamma) \cap \Omega$ and $\Omega \backslash \operatorname{int}(\gamma)$. This ends the proof of Theorem 4.1.

\section{Proof of Theorem 2.6}

In this section, we establish Theorem 2.6.

We will use the following notation: for $\delta>0$ and $E$ a subset of $\mathbb{R}^{p}, \mathcal{V}_{\delta}(E)$ will denote a neighborhood of thickness $\delta$ of $E$ :

$$
\mathcal{V}_{\delta}(E)=\{x \in \Omega / d(x, E)<\delta\}
$$

In the sequel, when referring to smooth curves, surfaces or maps, we mean of class $C^{\infty}$. We will also use these notions of curves, surfaces or maps in the real-analytic class, which we denote by $C^{\omega}$.

\subsection{A model flow}

The first part of the proof consists in introducing a model flow, that is a solenoidal vector field that drives $\gamma_{0}$ to $\gamma_{1}$ exactly. But of course, it does not necessarily satisfy (1.2).

For both cases $N=2,3$ the assumption that $\gamma_{0}$ and $\gamma_{1}$ are isotopic in $\Omega$ leads to the existence of a smooth isotopy preserving the volume given by the following theorem.

Theorem 5.1. Assume that $\gamma_{0}$ and $\gamma_{1}$ are isotopic in $\Omega$, there exists $X \in C_{c}^{\infty}\left([0,1] \times \bar{\Omega}, \mathbb{R}^{N}\right)$ such that $\operatorname{div}(X)=0$,

$$
\phi^{X}\left([0,1], 0, \gamma_{0}\right) \subset \Omega \text { and } \phi^{X}\left(1,0, \gamma_{0}\right)=\gamma_{1} .
$$

In the general situation, this is a direct consequence of a result of Krygin [19]. An explicit proof of Theorem 5.1 is given in [13] when $N=2$.

\subsection{Extension of analytic solutions of the Stokes equation across the boundary}

As in [13,14], an important case corresponds to the case when $\gamma_{0}$ and $X$ (such as given in Thm. 5.1) are real analytic data. The central point is that a solution of Stokes equation in an analytic domain with analytic data on the boundary can be extended across the boundary, which will allow to use Theorem 2.1.

Consider $\gamma$ a $C^{\omega}$ Jordan surface in $\Omega$ and $X$ a $C^{\omega}$ divergence free vector field on $\Omega$. Depending on $\Sigma$ and $\gamma$, we introduce a new open set $\widetilde{\Omega}(\gamma)$ as follows:

- If $\gamma$ is homotopic to a point in $\Omega$, we set $\widetilde{\Omega}(\gamma):=(\operatorname{int}(\gamma))^{\circ}$ (where we recall that $A^{\circ}$ refers to the topological interior of a subset $A$, and int $(\gamma)$ to the interior in the sense of Jordan's theorem). 
- If $\gamma$ is not homotopic to a point in $\Omega$ and if $\Sigma \subset \operatorname{int}(\gamma)$, we set $\widetilde{\Omega}(\gamma)=\Omega \backslash \operatorname{int}(\gamma)$.

- If $\gamma$ is not homotopic to a point in $\Omega$ and if $\Sigma$ meets $\partial \Omega \backslash \operatorname{int}(\gamma)$, we can always assume that $\Sigma \subset \partial \Omega \backslash \operatorname{int}(\gamma)$, and we set $\widetilde{\Omega}(\gamma):=\Omega \cap \operatorname{int}(\gamma)$.

In other words, according to the location of $\Sigma \subset \partial \Omega$ with respect to $\gamma, \widetilde{\Omega}(\gamma)$ denotes the interior or the exterior of $\gamma$ in $\Omega$.

We first recall the following result on the Stokes equation (see [24] or [8]).

Proposition 5.2. Let $\Omega$ a bounded smooth domain in $\mathbb{R}^{N}, N=2$ or 3 . For any $u_{0} \in H^{1 / 2}(\partial \Omega)^{N}$ such that

$$
\int_{\partial \Omega} u_{0} \cdot n \mathrm{~d} \sigma=0
$$

there exists a unique solution $(u, p) \in H^{1}(\Omega)^{N} \times L^{2}(\Omega)$ to (1.1) with $u=u_{0}$ on $\partial \Omega$.

The following proposition proves the possibility of extending analytic solutions across the boundary.

Proposition 5.3. Let $X \in C^{\omega}(\Omega)$ such that $\operatorname{div}(X)=0$ in $\Omega$. Let $(u, p)$ be a solution of (1.1a), (1.1b), (1.1c) in $\widetilde{\Omega}(\gamma)$ such that $u=X$ on $\gamma$, and such that $u_{\mid \partial \widetilde{\Omega}(\gamma) \backslash \gamma}=0$ (this condition can be empty). Then there exists a neighborhood $\mathcal{V}_{\delta}(\gamma)$ such that $(u, p)$ can be extended to a solution of (1.1a), (1.1b) in $\mathcal{V}_{\delta}(\gamma) \cup \widetilde{\Omega}(\gamma)$.

Proof of Proposition 5.3. This is a classical result. Indeed, for $N=2,3$, it is proven in [6] (see also [9] and [16]) that the Stokes systems satisfies the Agmon-Douglis-Nirenberg ellipticity conditions (see $[1,2]$.) Since $X$ is analytic and since $\gamma$ is analytic, the analyticity of $(u, p)$ in $\widetilde{\Omega}(\gamma)$ up to $\gamma$ is then classical (see [21], Sect. 6.6). This result also follows from the regularity results given in [16] and when $N=3$, one may use a similar argument as in [9].

We now follow the strategy given in [13].

\subsection{Proof of the main result when $X$ and $\gamma_{0}$ are analytic} that

We first assume that $\gamma_{0}$ is real analytic and that $X \in C_{c}^{1}\left([0,1] ; C^{\omega}(\Omega)\right)$. Due to the regularity of $X$, it follows

$$
\gamma(t):=\phi^{X}\left(t, 0, \gamma_{0}\right)
$$

defines a continuous family of real analytic surfaces. As in Proposition 5.3, we consider the solution $(u(t), p(t))$ of

$$
\left\{\begin{array}{l}
-\Delta u+\nabla p=0 \text { in } \widetilde{\Omega}(\gamma(t)) \\
\operatorname{div} u=0 \text { in } \widetilde{\Omega}(\gamma(t)) \\
u(t)=X(t) \text { on } \gamma(t) \\
u(t)=0 \text { on } \partial \widetilde{\Omega}(\gamma(t)) \backslash \gamma(t)
\end{array}\right.
$$

Then Proposition 5.3 determines some neighborhood $\mathcal{V}_{\delta(t)}(\gamma(t))$ of $\gamma(t)$ such that $(u, p)$ extends to $\mathcal{V}_{\delta(t)} \cup \widetilde{\Omega}(\gamma(t))$.

Let us now establish that, due to the analyticity and compactness of $\gamma_{0}$, one can obtain a uniform size $\delta(t)$ of the neighborhood $\mathcal{V}_{\delta(t)}(\gamma(t))$ in $[0, T]$ and moreover get that $u \in C\left([0,1] ; C^{\infty}\left(\widetilde{\Omega}(\gamma(t)) \cup \mathcal{V}_{\delta}(\gamma(t))\right)\right.$ with the abuse of notations that $C^{\infty}\left(\widetilde{\Omega}(\gamma(t)) \cup \mathcal{V}_{\delta}(\gamma(t))\right)$ actually depends on $t$. This means that $u$ is defined for each $t$ on $\widetilde{\Omega}(\gamma(t)) \cup \mathcal{V}_{\delta}(\gamma(t))$ and can be extended to a function in $C\left([0,1] ; C^{\infty}\left(\mathbb{R}^{N}\right)\right)$. First, since $\gamma$ and $X$ are continuous functions of time with values in real analytic functions in space, they can be chosen as the data in Proposition 5.3. Hence we can deduce from (the proof of) Proposition 5.3 that for each $(t, x)$ in $[0, T] \times \gamma$, there exist a neighborhood $U_{x}$ of $x$ and a neighborhood $\left(t-\eta_{t}, t+\eta_{t}\right)$ of $t$ such that $u$ can be extended in $U_{x}$ for all times in $\left(t-\eta_{t}, t+\eta_{t}\right)$. Using the compactness of $\gamma$ and the unique continuation principle, we can extend this 
to a whole neighborhood $\mathcal{V}_{\delta(t)}(\gamma(t))$ of $\gamma$ for times in $\left(t-\eta_{t}, t+\eta_{t}\right)$. Now given $\varepsilon>0$, using the compactness of $[0, T]$, we can obtain $0 \leq t_{1}<\cdots<t_{n} \leq 1, \eta_{t_{1}}, \ldots, \eta_{t_{n}}>0$ and $\delta>0$ such that

$$
\begin{gathered}
{[0,1] \subset \cup_{i=1}^{n}\left(t_{i}-\eta_{t_{i}}, t_{i}+\eta_{t_{i}}\right),} \\
\forall t \in\left[t_{i}-\eta_{t_{i}}, t_{i}+\eta_{t_{i}}\right] \cap[0,1], \gamma(t) \subset \mathcal{V}_{\delta / 2}\left(\gamma\left(t_{i}\right)\right), \\
\forall s, t \in\left[t_{i}-\eta_{t_{i}}, t_{i}+\eta_{t_{i}}\right], \quad\|u(s, \cdot)-u(t, \cdot)\|_{C^{k}\left(\overline{\mathcal{V}_{\delta / 2}}\left(\gamma\left(t_{i}\right)\right)\right)} \leq \varepsilon,
\end{gathered}
$$

and

$$
\forall(t, s) \in\left(t_{i}-\eta_{t_{i}}, t_{i}+\eta_{t_{i}}\right) \cap[0,1],\|X(t, \cdot)-X(s, \cdot)\|_{C^{k}\left(\mathcal{V}_{\delta / 2}\left(\gamma\left(t_{i}\right)\right)\right.} \leq \varepsilon .
$$

This includes in particular the uniformity that we claimed on the size $\delta(t)$ of the neighborhood $\mathcal{V}_{\delta(t)}(\gamma(t))$ in $[0, T]$. More details in the harmonic case can be found in ([13], Proof of Lem. 3).

Now for each $i$, we determine Jordan surfaces $\widehat{\gamma}_{i}$ in $\Omega \backslash \widetilde{\Omega}\left(\gamma\left(t_{i}\right)\right) \cap\left[\mathcal{V}_{3 \delta / 4}\left(\gamma\left(t_{i}\right)\right) \backslash \overline{\mathcal{V}}_{\delta / 2}\left(\gamma\left(t_{i}\right)\right)\right]$ such that $\gamma\left(t_{i}\right) \subset \widetilde{\Omega}\left(\widehat{\gamma}_{i}\right)$. The solution $\left(u\left(t_{i}\right), p\left(t_{i}\right)\right)$ can hence be extended up to $\widehat{\gamma}_{i}$.

We now apply Theorem 2.2 (one could use Thm. 4.1 as well). Given $\varepsilon>0$, we can find a solution $\left(U_{i}, P_{i}\right)$ of $(1.1)$ on $\Omega$ such that $U_{i \mid \partial \Omega} \in H_{m}^{1 / 2}(\partial \Omega ; \Sigma)$ and such that

$$
\left\|U_{i}-u_{i}\right\|_{H_{m}^{1 / 2}\left(\hat{\gamma}_{i}\right)}<\varepsilon \text {. }
$$

By classical elliptic estimates, we infer from (5.2) that, for any $k$, there exists $C$ (not depending on $\varepsilon$, but depending on $k, \widehat{\gamma}_{i}$ and $\delta$ ) such that

$$
\left\|U_{i}-u_{i}\right\|_{C^{k}} \overline{\left(\mathcal{V}_{\delta / 3}\left(\tilde{\Omega}\left(\gamma\left(t_{i}\right)\right)\right)\right.}<C \varepsilon
$$

Take $\left(\kappa_{i}\right)_{i=1 \ldots n}$ a smooth partition of unity associated to the covering $\left(t_{i}-\eta_{t_{i}}, t_{i}+\eta_{t_{i}}\right)_{i=1 \ldots n}$ and consider

$$
U(t, x):=\sum_{i=1}^{n} \kappa_{i}(t) U_{i}(x) \text { and } P(t, x):=\sum_{i=1}^{n} \kappa_{i}(t) P_{i}(x) .
$$

Then for all $t \in[0,1](U(t, \cdot), P(t, \cdot))$ is a solution of $(1.1)$, and we have

$$
\max _{t \in[0,1]}\|U-u\|_{C^{k}\left(\overline{\mathcal{V}_{\delta / 3}(\tilde{\Omega}(\gamma(t)))}\right)}<C \varepsilon,
$$

for some $C$ independent of $\varepsilon$. This implies that, provided $\varepsilon$ is small enough,

$$
\forall t \in[0,1],\|U\|_{C^{k}(\overline{\tilde{\Omega}(\gamma(t))})}<1+\|u\|_{C^{k}(\overline{\tilde{\Omega}(\gamma(t))})} .
$$

Note that $\phi^{u}\left(t, 0, \gamma_{0}\right)=\phi^{X}\left(t, 0, \gamma_{0}\right)$, and thus provided that $\varepsilon>0$ is small enough we have, by Gronwall's lemma

$$
\left\|\phi^{U}\left(t, 0, \gamma_{0}\right)-\phi^{u}\left(t, 0, \gamma_{0}\right)\right\|_{\infty} \leq\|u-U\|_{C^{0}\left([0,1] ; C^{0}\left(\overline{\mathcal{V}_{\delta / 3}}(\gamma(t))\right)\right)} \mathrm{e}^{\|u\|_{L^{1}\left(0,1 ; W^{1, \infty}\left(V_{\delta / 3}(\gamma(t))\right)\right)}},
$$

which proves in particular that the flow of $\gamma_{0}$ by $u$ remains in $\Omega$. By classical regularity and using again Gronwall's lemma and (5.4), we get for some $C$ independent of $\varepsilon$ :

$$
\left\|\phi^{U}\left(t, 0, \gamma_{0}\right)-\phi^{u}\left(t, 0, \gamma_{0}\right)\right\|_{C^{k}\left(\mathbb{S}^{N-1}\right)} \leq C\|u-U\|_{C^{0}\left([0,1] ; C^{k}\left(\overline{\mathcal{V}_{\delta / 3}}(\gamma(t))\right)\right)} \mathrm{e}^{\|u\|_{L^{1}\left(0,1 ; W^{1+k, \infty}\left(V_{\delta / 3}(\gamma(t))\right)\right)}},
$$

which ends the proof of Theorem 2.6 in this situation.

Remark 5.4. If one wants to use Theorem 4.1 rather than Theorem 2.2 to get the "extended approximation" $\left(U_{i}, P_{i}\right)$, one chooses $K=\overline{\mathcal{V}}_{\delta / 2}\left(\gamma\left(t_{i}\right)\right) \cup(\partial \Omega \backslash \Sigma)$ and one approximates the function given by $\left(u\left(t_{i}\right), p\left(t_{i}\right)\right)$ on $\mathcal{V}_{\delta / 2}\left(\tilde{\Omega}\left(\gamma\left(t_{i}\right)\right)\right)$ and by 0 on a neighborhood of $\partial \Omega \backslash \Sigma$. One does not get exactly $U_{i}=0$ on $\Sigma$, but one can remove a solution of (1.1) given by Proposition 5.3 and associated with boundary conditions given by $U_{i}$ on $\partial \Omega \backslash \Sigma$ and to an extension of $U_{i}$ on $\Sigma$ of comparable size. This way we obtain a suitable approximation satisfying moreover the boundary conditions on $\partial \Omega \backslash \Sigma$. 


\subsection{Proof of the main result when $X$ is analytic and $\gamma_{0}$ is smooth}

We will only consider the case of $N=3$, the case $N=2$ being similar. In this part we assume that $X$ is a solenoidal vector field in $C\left([0,1] ; C^{\omega}(\Omega)\right.$ ), and that $\gamma_{0}$ is a smooth (but not necessarily analytic) embedded 2 -sphere in $\Omega$.

According to a result of Whitney (see [25]), $\gamma_{0}$ is embedded in a smooth family of smooth Jordan surfaces $\gamma_{\nu}, \nu \in\left(-\nu_{0}, \nu_{0}\right)$, such that for $\nu \neq 0, \gamma_{\nu}$ is analytic, and for $\nu \neq \nu^{\prime}$ we have $\gamma_{\nu} \cap \gamma_{\nu^{\prime}}=\emptyset$. We can assume that $\gamma_{0} \subset \operatorname{int}\left(\gamma_{\nu}\right)$ for $\nu>0$.

Now for each $\nu>0$ and any $t \in[0,1]$, we consider $\left(u_{\nu}(t), p_{\nu}(t)\right)$ given by Proposition 5.3 with $\phi^{X}\left(t, 0, \gamma_{\nu}\right)$ instead of $\gamma$. Then given $\varepsilon>0$, we determine $\left(U_{\varepsilon, \nu}(t), P_{\varepsilon, \nu}(t)\right)$ such that $(2.5 \mathrm{a})$ holds with $\gamma_{\nu}$ instead of $\gamma_{0}$.

Let us point out that, using elliptic regularity (noting that the constant in the elliptic regularity estimate is uniform in $\nu$ due to $\gamma_{\nu} \rightarrow \gamma_{0}$ in $C^{\infty}$ as $\left.\nu \rightarrow 0\right)$, (5.1) and (5.5), we have uniform bounds with respect to $\nu$ on $\left\|U_{\varepsilon, \nu}\right\|_{C\left([0,1] ; C^{k}\left(\phi^{U_{\varepsilon, \nu}}\left(t, 0, \gamma_{\nu}\right)\right)\right.}$.

Therefore we get by Gronwall's lemma that

$$
\left\|\phi^{U_{\varepsilon, \nu}}\left(t, 0, \gamma_{\nu}\right)-\phi^{U_{\varepsilon, \nu}}\left(t, 0, \gamma_{0}\right)\right\|_{C^{k}\left(\mathbb{S}^{2}\right)} \leq C_{k}\left\|\gamma_{0}-\gamma_{\nu}\right\|_{C^{k}\left(\mathbb{S}^{2}\right)} \exp \left(\int_{0}^{1}\left\|U_{\varepsilon, \nu}\right\|_{C^{k+1}\left(\operatorname { i n t } \left(\phi^{\left.\left.U_{\varepsilon}\left(t, 0, \gamma_{\nu}\right)\right)\right)}\right.\right.} \mathrm{d} t\right)
$$

which proves the result in this situation.

\subsection{Proof of the main result in the general case}

Again we will consider the case $N=3$. We assume now that we are in the general case, that is, $X$ is merely $C^{\infty}\left(\bar{\Omega} ; \mathbb{R}^{3}\right)$. Let $\lambda>0$ such that

$$
\max _{t \in[0,1]} \operatorname{dist}\left(\phi^{X}\left(t, 0, \gamma_{0}\right), \partial \Omega\right)>2 \lambda .
$$

We define $\mathcal{U}_{t}:=\overline{\mathcal{V}}_{\lambda}(\operatorname{int}(\gamma(t)))$. Reducing $\lambda$ if necessary, we can obtain that for all $t, \mathcal{U}_{t}$ is diffeomorphic to a ball.

Now we can use Whitney's approximation theorem (see e.g. [18], Prop. 3.3.9): for any $\mu>0$ and any $k \in \mathbb{N}$ there exists $X_{\mu} \in C\left([0,1] ; C^{\omega}\left(\mathbb{R}^{3}\right)\right)$ such that

$$
\left\|X_{\mu}-X\right\|_{C\left([0,1] ; C^{k+1}\left(\mathcal{U}_{t}\right)\right)} \leq \mu .
$$

Moreover, we can ask that

$$
\operatorname{div} X_{\mu}=0 \text { in }[0,1] \times \mathbb{R}^{3} .
$$

This is just a matter of writing $X$ in the form $X=\operatorname{curl} A$ (using the fact that $\mathcal{U}_{t}$ is a topological ball) and approximating $A$ at order $k+2$.

Using as before the compactness of the time interval $[0,1]$ and a partition of unity (as in the proof when $X$ and $\gamma_{0}$ are analytic), we can obtain a smooth approximation uniformly in time.

We then apply Gronwall's lemma to infer that

$$
\left\|\Phi^{X}\left(t, 0, \gamma_{0}\right)-\Phi^{X}\left(t, 0, \gamma_{0}\right)\right\|_{C^{k}\left(\gamma_{0}\right)} \leq\left\|X-X_{\mu}\right\|_{C^{0}\left([0,1] ; C^{k}(\bar{\Omega})\right.} \exp \left(\|X\|_{L^{1}\left(0,1 ; C^{k+1}(\bar{\Omega})\right)}\right),
$$

and apply the preceding procedure when $\gamma_{0}$ is smooth and $X$ is analytic.

This concludes the proof of Theorem 2.6.

\section{A FINAL REMARK}

One may wonder if it is possible to take into account a non-trivial initial condition $(u, p)_{\mid t=0}=\left(u_{0}, p_{0}\right)$ at $t=0$, where $\left(u_{0}, p_{0}\right)$ is a solution of (1.1). The question is whether it is still possible to solve the problem of approximate Lagrangian controllability, that is, to find $(u, p)$ a time dependent solution of (1.1) such that (2.5a) holds and moreover satisfying $u_{\mid t=0}=u_{0}$ and $p_{\mid t=0}=p_{0}$. 
This is actually an easy consequence of Theorem 2.6. In order to do so, we assume that $\left(u_{0}, p_{0}\right)$ is a solution of (1.1) such that $u_{0} \in \mathcal{L} i p(\Omega)$. Consider $\tau>0$ such that for $t \in[0, \tau], \phi^{u_{0}}(t, 0, \tau) \subset \Omega$. Note that this is always possible due to the regularity of $u_{0}$. Define $\left(\widetilde{u}_{0}(t), \widetilde{p}_{0}(t)\right)=\frac{(\tau-t)^{2}}{\tau^{2}}\left(u_{0}, p_{0}\right)$ then $\forall t \in[0, \tau], \phi^{\widetilde{u}_{0}}\left(t, 0, \gamma_{0}\right) \subset \Omega$ and we have $\widetilde{u}_{0}(\tau)=0$. We can then apply the same procedure as before and obtain

Theorem 6.1. The results of Theorem 2.6 remain true when we impose $u_{\mid t=0}=u_{0} \in \mathcal{L}$ ip $(\Omega)$, with the constraint that $\operatorname{div} u_{0}=0$ and $u_{0 \mid \partial \Omega \backslash \Sigma}=0$.

Acknowledgements. The authors wish to thank the anonymous referees for their careful reading of the paper and their many suggestions and corrections. TH wishes to thank M. Costabel for fruitful indications on some regularity issues. OG is partially supported by ANR Project IFSMACS (ANR-15-CE40-0010).

\section{REFERENCES}

[1] S. Agmon, A. Douglis and L. Nirenberg, Estimate near the boundary for the solutions of elliptic differential equations satisfying general boundary values i. Comm. Pure Appl. Math. 12 (1959) 623-727.

[2] S. Agmon, A. Douglis and L. Nirenberg, Estimate near the boundary for the solutions of elliptic differential equations satisfying general boundary values ii. Comm. Pure Appl. Math. 17 (1964) 35-92.

[3] F. Alouges and L. Giraldi, Enhanced controllability of low reynolds number swimmers in the presence of a wall. Acta Appl. Math. 128 (2013) 153-179.

[4] F. Alouges, A. DeSimone and A. Lefebvre, Swimming at low Reynolds number at optimal strokes: An example. J. Nonlin. Sci. 3 (2008) 277-302.

[5] J.-B. Biot and F. Savart, Note sur le magnétisme de la pile de volta. Ann. Chim. Phys. (1820) 1222-1223.

[6] P.B. Bochev and M.D. Gunzburger, Least-squares methods for the velocity-pressure-stress formulation of the stokes equations. Comp. Meth. Appl. Mech. Eng. 126 (1995) 267-287.

[7] M. Boulakia, A.-C. Egloffe and C. Grandmont, Stability estimates for a Robin coefficient in the two-dimensional Stokes system. Math. Control Relat. Fields 3 (2013) 21-49.

[8] F. Boyer and P. Fabrie, Eléments d'analyse pour l'étude de quelques modèles d'écoulements de fluides visqueux incompressibles. In vol. 52 of SMAI, Mathématiques et Applications. Springer-Verlag, Berlin, Heidelberg (2005).

[9] M. Costabel, M. Dauge and S. Nicaise, Analytic regularity for linear elliptic systems in polygons and polyhedra. Math. Models Methods Appl. Sci. 22 (2012) 1250015.

[10] D.B.A. Epstein, Curves on 2-manifolds and isotopies. Acta Math. 115 (1966) 83-107.

[11] C. Fabre and G. Lebeau, Prolongement unique des solutions de l'equation de stokes. Commun. Partial Differ. Eq. 21 (1996) $573-596$.

[12] S.J. Gardiner, Harmonic approximation. Vol. 221 of London Mathematical Society, Lecture Note Series. Cambridge university press (1995).

[13] O. Glass and T. Horsin, Approximate lagrangian controllability for the 2-d Euler equations. Application to the control of the shape of vortex patch. J. Math. Pures Appl. 93 (2010) 61-90.

[14] O. Glass and T. Horsin, Prescribing the motion of a set of particles in a 3d perfect fluid. Soumis (2011).

[15] P.W. Gross and P.R. Kotiuga, Electromagnetic theory and computation: a topological approach. Vol. 48 of Mathematical Sciences Research Institute Publications. Cambridge University Press, Cambridge (2004).

[16] B. Guo and C. Schwab, Analytic regularity of stokes flow on polygonal domains in countably weighted sobolev spaces. J. Comput. Appl. Math. 190 (2006) 487-519.

[17] E. Guyon, J.-P. Hulin and L. Petit, Hydrodynamique physique, 3ème édition. Savoirs actuels. EDP Sciences/CNRS Éditions, 3rd edition (2012).

[18] S.G. Krantz and H.R. Parks, A Primer of Real Analytic Functions. Birkhäuser, Basel, Boston, Berlin (1992).

[19] A.B. Krygin, Extension of diffeomorphisms that preserve volume. Funkcional. Anal. i Priložen. 5-2 (1971) 72-76.

[20] J. Lohéac and A. Munnier, Controllability of 3D low Reynolds number swimmers. ESAIM COCV 20 (2014) $236-268$.

[21] C.B. Morrey, Multiple integrals in the calculus of variations. Classics in Mathematics. Reprint of the 1966 edition [MR0202511]. Springer-Verlag, Berlin (2008).

[22] W. Rudin, Real and complex analysis. McGraw-Hill Series in Higher Mathematics. McGraw-Hill Book Co., New York, 2nd edition (1974).

[23] J. San Martin, T. Takahashi and M. Tucsnak, Théorie du mouvement non permanent des eaux, avec applications aux crues des rivières et à l'introduction des marées dans leur lit. Quart. Appl. Math. 65 (2007) 405-424.

[24] R. Temam, Navier-Stokes Equations: Theory and numerical analysis. North-Holland Publications, North-Holland (1979).

[25] H. Whitney, The imbedding of manifolds in families of analytic manifolds. Ann. Math. 37-4 (1936) 865-878. 